\title{
Sistematización de las experiencias del curso en línea "Nuevas tecnologías y Terapia Ocupacional": Nuevas competencias para la Terapia Ocupacional
}

\section{Systematization of the experiences of the course on line "New Technologies and Occupational Therapy": New Competences for Occupational Therapy}

\author{
Jaime Fagnilli G. ${ }^{1}$, Lisette Farias V. ${ }^{2}$
}

\section{Resumen}

Este articulo sistematiza diferentes aspectos del desarrollo, implementación y resultados del curso "Las Nuevas Tecnologías y Terapia Ocupacional", el primer curso on-line en Chile, creado por REHABCHILE, orientado a estudiantes y Terapeutas Ocupacionales. Este curso tuvo como propósito que los participantes pudiesen aprender acerca de lo que actualmente se conoce como Tecnologías de Información y Comunicación, y explorar sus posibles usos a través de actividades grupales, relacionadas con el quehacer de la Terapia Ocupacional.

La metodología de enseñanza utilizada en este curso fue el "Aprendizaje Colaborativo" y el modo de trabajo fue on-line. El curso tuvo una duración de 5 meses, constó con tres módulos de trabajo y 21 participantes, los cuales se dividieron en cinco grupos. Los resultados de éste curso muestran un grado de satisfacción positivo de los participantes con la modalidad de trabajo utilizado y con las herramientas tecnológicas exploradas. Además, los resultados de este curso demuestran que existe un número creciente de profesionales y estudiantes que están interesados en conocer los usos y beneficios de las nuevas tecnologías en el contexto de la Terapia Ocupacional.

En conclusión, la sociedad está siendo testigo de una nueva era de la información y la comunicación, la cual está influyendo decisivamente en las actividades de la vida diaria de miles de personas. Es por esto que la Terapia Ocupacional debe acercarse a las nuevas tecnologías para evaluar las oportunidades que estas ofrecen para facilitar el debate, la construcción del conocimiento y el desarrollo profesional, lo mas pronto posible.

Palabras clave: Tecnologías de Información y Comunicación, Terapia Ocupacional y Aprendizaje Colaborativo.

\footnotetext{
Abstract

This article systematizes different aspects of development, implementation and outcomes of the course "New Technologies and Occupational Therapy", the first on-line course in Chile,

${ }^{1}$ Terapeuta Ocupacional, Bachelor Degree in Occupational Therapy, Universidad de Chile. HealthCare Industry. Dirección Postal: 9266 -209 University Crescent, Burnaby BC V5A 4Z1 Canada: Fono: 778 9905966 Mail: fagnillij@gmail.com

2 Terapeuta Ocupacional, Lic. en Cs. de la Ocupación Humana, Universidad de Chile. Estudiante European Master of Science in Occupational Therapy. Dirección Postal: Orvar Odds Väg 51, 11254 Estocolmo, Suecia. Fono: 5709486 (Chileno) Mail: lissette.farias@gmail.com
} 
created by REHABCHILE and directed towards Occupational Therapy students and professionals. This course had as purpose that participants could learn about what is currently known as Information and Communication Technologies, and explore its possible uses through group activities related to the practice of Occupational Therapy.

The methodology of teaching used in this course was the "Collaborative Learning" and the type of work was on-line. The course lasted 5 months, consisted of three modules of work and had 21 participants who were divided into five groups. The results of this course reveal a positive level of participant satisfaction with the working method used and the technological tools explored. Furthermore, the results of this course demonstrate that there is a growing number of professionals and students who are interested in the uses and benefits of new technologies in the context of Occupational Therapy.

In conclusion, society is witnessing a new era of information and communication, which is decisively influencing the activities of the daily lives of thousands of people. This is why Occupational Therapy should approach these new technologies to evaluate the opportunities that they offer to facilitate discussion, knowledge building and professional development, as soon as possible.

Key words: Information and Communication Technologies, Occupational Therapy and Collaborative Learning.

\section{Introducción}

Las Nuevas Tecnologías o Tecnologías de Información y Comunicación (TIC), son un término que se usa para referirse a una categoría especial de software o programas utilitarios disponibles en Internet para cualquier usuario que esté interesado en ocupar éstas herramientas. Estos softwares se utilizan en dispositivos electrónicos tales como; celulares, computadores, tablets, entre muchos otros existentes en el mercado hoy en día (1).

Desde su introducción, las TIC también han sido llamadas "Sitios de Redes Sociales (SRS)" o "Social Network Sites (SNSs)", siendo algunos ejemplos de éstos; Facebook, Twitter, MySpace, Flicker, Picassa, Youtube, Google Docs, Foros, Chats, Messenger, Skype, Wikis, Blogs y Podcast, siendo los tres últimos también llamados "Herramientas Web 2.0" o "Webbased collaborationware" (2). Estas nuevas tecnologías han atraído a millones de usuarios, muchos de los cuales han integrado éstos sitios a sus prácticas de uso diario, convirtiéndolas en parte esencial de sus rutinas (3-6). El impacto de las TIC es tal, que en las últimas décadas éstas han mejorado ostensiblemente las capacidades comunicacionales, tanto que hoy en día, la literatura señala que los Wikis, blogs/fotoblogs y Podcast tienen el potencial de complementar, mejorar y añadir nuevas dimensiones de colaboración e intercambio de información; en la educación basada en la Web, en el desarrollo profesional continuo y en los servicios de investigación existentes en la actualidad (2).

Debido al innegable efecto social de las TIC en el quehacer diario de las personas en todo el mundo, investigadores de distintos campos han examinado las TIC con el fin de comprender las prácticas de sus usos, consecuencias, lenguaje, cultura y significado de éstos sitios, así como su popularidad y la motivación de los usuarios por participar en ellos (5-7). 
Desde la Terapia Ocupacional (TO), este fenómeno amerita una mirada tomando como referente nuestra disciplina, ya que no solo representa un aspecto importante en la vida diaria de la población, creando grupos on-line basados en aspectos comunes como; intereses, opiniones políticas, actividades, lenguaje, identidad religiosa y/o étnica (5), sino que además las TIC han pasado a ser parte relevante del quehacer de nuestra sociedad, facilitando la obtención de información, conocimiento y debate, a través de la colaboración virtual. Con el uso de estas tecnologías es posible compartir diálogo e información entre participantes de proyectos en grupo, o posibilita a los estudiantes y/o profesionales a participar en el aprendizaje de unos con otros, utilizándolas como un entorno de colaboración para la construcción de conocimientos o para ser parte de una comunidad de práctica virtual (2).

Además, si tomamos la definición de ocupación como todas aquellas tareas y actividades en las cuales un individuo o grupo se involucra diariamente y que son cultural y personalmente significativas $(8,9)$, podríamos establecer entonces, que el uso de nuevas tecnologías podría ser considerado como una ocupación más del ser humano debido a que éstas constituyen una parte importante de la vida diaria, creando significado y un lenguaje e interés común para cientos de personas.

Por otra parte, podemos reforzar esta idea demostrando que el uso de estas tecnologías se hace evidente en las distintas áreas de la ocupación, tales como el área del trabajo o productividad, donde podemos observar el uso de las TIC para efectuar cursos de desarrollo profesional continuo, videoconferencias, educación a distancia, etc. En el área de esparcimiento o tiempo libre, encontramos su uso en personas que se conectan a través de tecnologías tales como Messenger, correos electrónicos, Twitter, Facebook, Fotoblogs, Wikis, etc. En el área de las actividades de autocuidado, podemos encontrar evidencia de su uso en actividades para facilitar la movilidad funcional sustentándose en el uso de aplicaciones computacionales o de móviles para revisar los horarios del transporte público, realizar transacciones bancarias y pago de cuentas de servicios básicos.

Siendo los Terapeutas Ocupacionales (TTOO), profesionales de la salud que prestan apoyo a una amplia gama de personas durante todas las etapas de la vida, es imprescindible incluír hoy en día, la utilización de soporte tecnológico para la realización de actividades relacionadas con los intereses personales de los usuarios. Del mismo modo, los TTOO se desempeñan en una variedad de escenarios, los que pueden representar retos tecnológicos o posibilidades para desarrollar programas apoyados por las nuevas tecnologías. Sin embargo, la literatura señala que la inserción de conocimientos y uso de las nuevas tecnologías, se ha limitado a carreras como Enfermería y Medicina y aún no se ha extendido a otras profesiones de la salud como la Terapia Ocupacional (10). Un ejemplo de esto, es que en la actualidad, sólo un número limitado de TTOO están involucrados en el área de los blogs científicos, existiendo evidencia que sugiere que esto se debe a la falta de conciencia de los beneficios potenciales de los blogs, los conceptos erróneos sobre el nivel de capacidad tecnológica necesaria y la preocupación por la exposición pública (11). Esto también puede obedecer a una falta de evidencia empírica que apoye a los blogs como una herramienta para el desarrollo profesional y a una renuencia a invertir tiempo por parte de los TTOO, en formar parte de la comunidad de TO on-line (11).

Un ejemplo del avance de las aplicaciones de las TIC en la educación en salud, es el crecimiento de los métodos de enseñanza on-line en la carrera de Enfermería en Australia y Nueva Zelanda (12-17), donde se están utilizando actividades que apoyan a los estudiantes a explorar, aprender y pensar on-line a través del uso de técnicas de interrogatorio, ejercicios y ejemplos de aplicaciones clínicas, las cuales son presentadas en una serie de interconexiones 
de páginas Web organizadas por temas (18). La literatura también señala que por ej. los Postcast están siendo utilizados en escuelas de Medicina alrededor del mundo, en donde se aprovechan las cualidades educativas de podcasting y videocasting, para grabar conferencias para aquellos estudiantes que por alguna eventualidad no pudieron asistir o para traspasar el contenido de textos de estudio en audio y permitirle a los alumnos "escucharlos", mientras se desplazan de un lugar a otro. $(19,20)$.

En Chile, la primera instancia de exposición de las nuevas tecnologías en relación con la Terapia Ocupacional, se produjo durante el transcurso del Congreso Mundial de TO, realizado en Chile en Mayo del 2010, en donde los integrantes de la agrupación OT4OT ("Online Tecnology for Occupational Therapy" o traducido al español; "Tecnología en Línea para la Terapia Ocupacional") expusieron sobre ésta materia, mostrando las diferentes aplicaciones de éstas nuevas tecnologías en áreas diversas, explicitaron sus experiencias y alentaron a los participantes a involucrarse mas activamente.

Debido a la carencia de instancias de aprendizaje on-line relacionadas con la $\mathrm{TO}$ en Chile, REHABCHILE decide introducir dentro de sus actividades educacionales, el primer y único curso on-line, llamado "Nuevas tecnologías y Terapia Ocupacional", enfocado a entregar una aproximación básica de la temática de las Nuevas Tecnologías a TTOO, profesionales y estudiantes, con el objetivo de satisfacer la necesidad de éstos por conocerlas, explorar sus usos y enriquecerse con los beneficios optimizando el quehacer de la TO en nuestro país. Esto se realiza, dentro del marco de un ambiente educacional, apoyado por la metodología de enseñanza llamada "Aprendizaje Colaborativo" $(21,22)$ la que destaca las ventajas que tiene el aprendizaje grupal, sobre el aprendizaje individual ya que el aprendizaje colaborativo al ser un proceso social de construcción del conocimiento, permite a los participantes aprender según los propios intereses y tiempo, apoyándose en el supuesto que, en el trabajo colaborativo se aprende más de lo que podría aprender individualmente.

Cabe destacar que REHABCHILE, es un programa de podcasting, el cual tiene un sitio en internet dirigido a cooperar con la formación de TTOO y otros profesionales de la salud, dirigiéndose principalmente a la entrega de contenidos relacionados con la TO a través de la modalidad de Podcast, donde se discuten distintas temáticas a través de entrevistas y foros de discusión para facilitar el desarrollo, colaboración y expansión del conocimiento de nuestra disciplina.

En conclusión, el curso "Nuevas tecnologías y Terapia Ocupacional", se gestó para generar una aproximación hacia el fenómeno tecnológico de las TIC y las actividades que éstas conllevan. Los autores de este artículo consideran necesaria la exploración de sus usos, beneficios y aplicaciones relacionados al quehacer educativo, administrativo y de la práctica de la Terapia Ocupacional en Chile.

\section{Marco Teórico}

En la historia de la humanidad el trabajar y aprender juntos ha sido vastamente difundido, aunque solo a fines del siglo XX surge el concepto de "Aprendizaje Colaborativo" o también llamado "Trabajo colaborativo", el cual se transformó en un tema de estudio en torno al cual se ha teorizado ampliamente (22). El "Aprendizaje Colaborativo" se refiere a una metodología didáctica que permite un proceso social de construcción del conocimiento $(21,22)$. Otras definiciones de Aprendizaje colaborativo añaden a esta definición; la adquisición de destrezas 
y actitudes que ocurren como resultado de la interacción en grupo (23), la noción de construcción del consenso, a través de la cooperación de los miembros del grupo (24) y el compromiso de las partes a aprender algo en conjunto (25).

Cabe destacar que el Aprendizaje colaborativo tiene como marco teórico al Constructivismo sociocultural que afirma que todo aprendizaje es social y mediado, el cual es congruente con la teoría Conversacional de Pask (26), la cual sigue el punto de vista de Vygotsky (27), quien postula que el hecho de aprender es por naturaleza un fenómeno social, en el cual la adquisición del nuevo conocimiento es el resultado de la interacción de gente que participa en un diálogo. De hecho, el aprendizaje colaborativo postula que aprender es un proceso dialéctico y dialógico en el que un individuo contrasta su punto de vista personal con el de otro hasta llegar a un acuerdo. Ese otro, también puede ser un "sí mismo", de esta forma incluímos el dialogo íntimo y personal consigo mismo (23).

En cuanto al término "Aprendizaje colaborativo", éste se ha desarrollado y gestado a través de distintas vertientes que buscan aproximarse a su significado. Así, la literatura nos presenta este término en relación a; los grupos de aprendizaje o learning groups, comunidades de aprendizaje o learning comunities, enseñanza entre pares o peer teaching, aprendizaje cooperativo cooperative learning, y aprendizaje colaborativo o collaborative learning $(23,25$, 28).

La razón por la que el curso de "Nuevas Tecnologías y Terapia Ocupacional" eligió utilizar el Aprendizaje colaborativo en su marco teórico, tiene relación con la importancia y con la velocidad que ha alcanzado el desarrollo del entorno de aprendizaje digital generado por la red de Internet. Este nuevo entorno electrónico de aprendizaje, que antes no existía, y que pone en contacto simultáneamente a millones de personas a través de redes digitales, ha creado un contexto concreto en el que se puede articular el carácter colaborativo del aprendizaje. Desde este punto de vista, a través de las TIC se validan las interacciones sociales, como también la visión de que el aporte de dos o más individuos que trabajan en función de una meta común, puede tener como resultado un producto más enriquecido y logrado, que la propuesta de uno sólo, esto favorecido por las interacciones, negociaciones y diálogos que dan origen al nuevo conocimiento.

En este contexto, el curso de "Nuevas Tecnologías y TO" considera esta nueva plataforma educacional del siglo XXI, un lugar en donde se puede facilitar el trabajo y aprendizaje colaborativo de un gran grupo de personas y profesionales del área de la Salud. Por consiguiente, el proyecto educativo aquí presentado pretende mostrar que la actividad colaborativa es posible a través de la Internet y que el aprendizaje colaborativo, está centrado básicamente en el diálogo y la negociación entre los integrantes del grupo de trabajo.

Para lograr lo anterior, nos adscribimos en este curso a los lineamientos de Driscoll y Vergara (29) sobre Aprendizaje colaborativo, donde se entiende que para que exista un verdadero aprendizaje colaborativo, no sólo se requiere trabajar juntos, sino que además cooperar en el logro de una meta que no se puede lograr individualmente. Además, se incluyeron en este curso, los siguientes cinco elementos que caracterizan el aprendizaje colaborativo:

1) Responsabilidad individual: todos los miembros son responsables de su desempeño individual dentro del grupo.

2) Interdependencia positiva: los miembros del grupo deben depender los unos de los otros para lograr la meta común. 
3) Habilidades de colaboración: las habilidades necesarias para que el grupo funcione en forma efectiva, como el trabajo en equipo, liderazgo y solución de conflictos.

4) Interacción promotora: los miembros del grupo interactúan para desarrollar relaciones interpersonales y establecer estrategias efectivas de aprendizaje.

5) Proceso de grupo: el grupo reflexiona en forma periódica y evalúa su funcionamiento, efectuando los cambios necesarios para incrementar su efectividad.

Para poner en práctica el aprendizaje colaborativo, este curso utilizó tres tipos de modalidades, las cuales integran los cinco elementos ya descritos, estas modalidades son; (a) Tareas Grupales, (b) Dinámicas Grupales y (c) Niveles Personales.

(a) La literatura ha demostrado que el trabajo colaborativo se ve potenciado a través de Tareas Grupales, ya que con éstas se logran objetivos de mayor riqueza cualitativa ya que reúnen propuestas y soluciones de varias personas del grupo, aumentando el aprendizaje y responsabilidad de cada participante, con lo que se enriquece la experiencia de aprender, aumentando las habilidades de colaboración y la motivación individual y grupal.

(b) La inclusión de Dinámicas grupales en este curso se justifica porque promueven la cercanía entre los participantes, su interdependencia positiva e interacción promotora, además de incrementar la satisfacción por el propio trabajo y valorar a sus pares.

(c) Asimismo, el trabajo a Nivel personal de los participantes fomenta la reflexión individual y la reflexión grupal través de las discusiones de sus vivencias durante el curso, aumentando las habilidades sociales, de interacción y comunicación afectivas, disminuyendo la individualidad y el temor a la crítica, aumentando la retroalimentación, incentivando el pensamiento crítico y reforzando la autoestima de los participantes.

En conclusión y en congruencia con lo descrito anteriormente, el propósito principal del curso de las "Nuevas Tecnologías y Terapia Ocupacional" fue incrementar en los participantes; el uso de la metodología de Aprendizaje Colaborativo on-line para el entendimiento de lo que actualmente se conoce como "Nuevas Tecnologías", en relación a sus aplicaciones concretas en las distintas áreas de la Terapia Ocupacional.

Los objetivos específicos de este curso fueron:

- Conocer la historia y el estado del arte de las TIC o Nuevas Tecnologías.

- Explorar la utilización de las TIC dentro del marco de la práctica de la TO.

- Identificar elementos efectivos de estas tecnologías en las distintas áreas de la práctica de la TO, focalizándose en las potencialidades relacionadas con el proceso de enseñanza / aprendizaje, inherente al quehacer diario de los TTOO.

- Explorar la utilización de estas tecnologías como herramientas administrativas, de evaluación e intervención terapéutica de forma secundaria.

\section{Metodología}

El diseño elegido para este curso fue construido en base a los formatos de cursos on-line, el cual le otorga a los participantes la posibilidad de interactuar entre ellos virtualmente, aportando al trabajo en grupo desde el lugar en que estén situados y con la disponibilidad de tiempos individuales. 
Los participantes de este curso fueron contactados y posteriormente invitados a participar al curso vía on-line, a través del uso de la página de REHABCHILE (18) en Facebook y Twitter. Además se enviaron invitaciones a participar vía correo electrónico a personas individuales y organizaciones relacionadas con Terapia Ocupacional en Chile. Los criterios de selección para acceder a este curso fueron: 1) Acreditar calidad de estudiante o profesional de Terapia ocupacional y 2) Poseer conocimiento básico en el uso de computador.

En cuanto a los requerimientos técnicos básicos para participar, éstos fueron: a) tener acceso a un computador con conexión a Internet con fonos o parlantes y micrófono (integrado o externo) y b) tener softwares instalados de descarga y uso gratuito en el computador, como Browser: Safari o Firefox, PDF reader, Video Player (Quick Time, WM Player, VLC) y Skype. Las herramientas tecnológicas exploradas en este curso fueron: Facebook, Google Docs, Skype, Wiki, Blogs, Google presentación, Google calendar y Google hoja de cálculo.

El número de interesados en participar, inicialmente fue alto. Los potenciales participantes fueron 29 individuos, los cuales respondieron principalmente a la invitación a través de la página de REHABCHILE en Facebook. Con este grupo inicial, todos relacionados con la disciplina de Terapia Ocupacional, se conformaron cinco grupos; tres grupos de cinco personas cada uno y dos grupos de siete integrantes. Sin embargo, de los 29 participantes inscritos al comienzo, 8 participantes desertaron antes de completar el primer modulo, quedando 21 participantes, de los cuales solo 17 de ellos, completaron el modulo dos y solo 15 completaron el modulo tres (Para datos generales de los participantes, Ver Tabla 1).

\begin{tabular}{|l|c|c|c|c|}
\hline Profesión & $\begin{array}{l}\text { Años de experiencia o } \\
\text { estudios de TO (Rango) }\end{array}$ & Mujeres & Hombres & Total N (\%) \\
\hline $\begin{array}{l}\text { Terapeutas } \\
\text { Ocupacionales }\end{array}$ & 5 meses - 15 años & 12 & 2 & $14(67 \%)$ \\
\hline $\begin{array}{l}\text { Estudiantes de Terapia } \\
\text { Ocupacional }\end{array}$ & 4to año - 5to año & 4 & 3 & $7(33 \%)$ \\
\hline Total participantes & & 16 & 5 & 21 \\
\hline
\end{tabular}

Tabla 1 Datos generales de los participantes

Este curso se realizó entre los meses de Abril-Agosto 2010 y constó con tres módulos de trabajo. Las horas de trabajo por participante, dependió principalmente del involucramiento y desarrollo personal de cada uno de éstos, calculándose un promedio de entre 40-60 horas de trabajo individual por módulo.

Previo al inicio de las actividades propias del curso, se envió a cada participante vía mail un cuestionario inicial evaluativo para determinar los conocimientos de estos en relación a: a) el concepto de las nuevas tecnologías, b) nuevas tecnologías que los participantes conocen y utilizan en su quehacer diario, c) el propósito o los objetivos por los cuales los participantes utilizan éstas tecnologías y d) las expectativas que ellos tenían de este curso. Además, se envió a cada alumno, documentos con información en cuanto a: 1) modalidad de trabajo, 2) contenido del programa y 3) bases y principios que se utilizarían en el desarrollo de este curso. 
Las asignaciones o trabajos entregados por los participantes, fueron evaluados con los siguientes parámetros: a) Creatividad y b) Profundización de los temas, con una escala de 1 al 4 (1: pobre; 2: Suficiente; 3: Bueno; 4: Sobresaliente), solo a modo de referencia, ya que este curso no fue concebido para obtener una certificación en la temática a tratar, sino que solo pretendió fomentar el uso y conocimiento tecnológico relacionado a la TO. Cabe destacar, que al término del curso se le envió a cada participante una encuesta on-line para evaluar el grado de satisfacción con el curso y además se les permitió guardar los materiales entregados, al igual que trabajos y presentaciones para futuros usos en sus prácticas diarias.

En general, los módulos de trabajo requerían de un trabajo on-line, utilizando los ambientes creados para este efecto. A modo de ejemplo se confeccionaron documentos en Google Docs para que los grupos, en cada modulo pudiesen desarrollar las actividades asignadas en esta plataforma de acceso gratuito. Otros ejemplos de ambientes utilizados fueron: Google presentaciones, Google planilla de cálculo, etc., sin perjuicio de la utilización de otras modalidades y/o plataformas on-line.

Los contenidos de los módulos estuvieron directamente relacionados con los objetivos del curso. Estos contenidos o materiales de estudio, fueron diseñados para que la intercomunicación entre profesor y alumnos fuese expedita y accesible. Los recursos utilizados como materiales de estudio, fueron en su mayoría en formato multimedia, tales como: Artículos de Internet, Sitios de Internet, Fotos, Vídeos, Presentaciones en Power Point, Podcast, VideoCasts, Blogs, Screencast, etc. (el material en Inglés fue de lectura opcional).

Los módulos, tuvieron como objetivo general que los participantes, al finalizar el curso estuvieran capacitaos para responder a las siguientes preguntas como resultado de su proceso de trabajo colaborativo y reflexiones personales:

- ¿Cuál es la relación que existe entre un grupo de aplicaciones computacionales y la Ocupación Humana?

- ¿Cuál es la importancia de las nuevas tecnologías en la sociedad actual?

- ¿Como afectan o influyen en la ejecución de otras actividades humanas?

- ¿Por que la Terapia Ocupacional debe prestar atención al tema de las Nuevas Tecnologías?

A continuación se describirá cada modulo con sus objetivos y actividades a modo de ejemplificar las actividades llevadas a cabo en este curso:

\section{Modulo I Historia y estado del Arte de las Nuevas Tecnologías}

Los objetivos de este módulo fueron, que el alumno al finalizar el curso estuviera en condiciones de:

- Conocer los principios del proceso de Aprendizaje colaborativo.

- Vivenciar el trabajo colaborativo a través de un aporte individual para la creación de material escrito en conjunto con su grupo de trabajo.

- Explorar el uso de "Google Docs" como una de las herramientas que ofrece las "Nuevas Tecnologías".

Asignación de actividades individuales:

1. Revisar el documento "Modulo I: Historia y Estado del Arte de Las Nuevas Tecnologías".

2. Revisar el documento "Bases y Principios usados en este curso".

3. Revisar material de estudio asociado al "Modulo I", a través de los links que se encuentran en ese documento. 
Asignación de Trabajo grupal:

1. Crear material escrito del tema de los "Blogs" en mayor profundidad.

2. Crear material escrito con los protocolos de actividades o pasos necesarios para abrir una cuenta en un sitio de Blog gratuito.

3. Crear material escrito con los primeros "post" de un blog, con el relato de la experiencia vivida de su primer trabajo colaborativo.

\section{Modulo II Exploración de las Nuevas Tecnologías}

Los objetivos de este modulo fueron que el alumno al finalizar el curso estuviera en condiciones de:

- Auto-capacitarse en el uso de manuales para la creación de material educativo utilizando otras herramientas que ofrece Google Docs y Skype.

- Poner en práctica esta auto-capacitación durante la creación y exposición de una presentación virtual.

- Vivenciar el trabajo colaborativo a través de un aporte individual para la creación de una exposición de diapositivas en el ambiente de presentaciones de Google.

- Vivenciar una clase a distancia modificada, a través del uso de las herramientas: "Skype" y "Google Docs".

Asignación de actividades individuales:

1. Revisar el documento "Modulo II: Manual de uso de Skype y Google Docs" y el material asociado a éste, a través de los links que se encuentran en éste documento.

Asignación de Trabajo grupal:

1. Crear un material escrito en relación a los WIKI: focalizado al ámbito de la educación y el quehacer de los TTOO (Historia, características, pasos para abrir cuenta, ej. de wiki en la educación, análisis de los elementos característicos de los wikis utilizables en la TO).

2. Crear presentación con el tema WIKI la cual será presentada vía Skype y Google simultáneamente. Esta presentación debe contener un análisis que relacione a los wikis con el quehacer de los TTOO (donde, como y por que los wikis podrían ser utilizados en el quehacer o práctica clínica de los TTOO). Agendar e inscribir la presentación en calendario Google del curso.

\section{Modulo III Aplicación de las Nuevas Tecnologías}

Los objetivos de este módulo fueron que el alumno al finalizar el curso estuviera en condiciones de:

- Explorar el uso de "Google Docs" como una de las herramientas que ofrece las "Nuevas Tecnologías".

- Auto-capacitarse en el uso de manuales para la creación de material educativo utilizando otras herramientas que ofrece Google Docs y Skype.

- Vivenciar la producción de un Podcast educativo.

- Explorar las fortalezas y debilidades de Google Calendar, Google Hoja de Cálculo y Google presentaciones.

Asignación de Trabajo grupal:

1. Enumerar las actividades administrativas de un TTOO: Por cada actividad determinar cual o cuales herramientas dentro de las Nuevas Tecnologías, podrían potencialmente facilitar esa actividad y fundamentar. 
2. Crear un sistema de horario de servicios de Terapia con Google Calendar (horas dadas a pacientes para sus sesiones de TO)

3. Crear un sistema de seguimiento de pacientes utilizando Google hoja de cálculo.

4. Enumerar las actividades de educación de un TTOO en la práctica diaria mencionando cual o cuales herramientas dentro de las Nuevas Tecnologías, podrían potencialmente facilitar esa actividad y fundamentar.

5. Crear un proyecto o protocolo para llevar a cabo solo una de las actividades mencionadas, incluyendo una o más de las herramientas exploradas y mencionar las características del grupo objetivo, el propósito general, los objetivos a alcanzar, las herramientas a utilizar, el tiempo presupuestado, etc., y por ultimo finalizar con una justificación de las herramientas elegidas para implementar este proyecto o plan de acción.

6. Crear una presentación on-line en google con el material elaborado para las asignaciones de este módulo.

\section{Resultados}

Los resultados presentados se basan en el análisis de los datos recabados a través de la encuesta on-line de satisfacción de los participantes del curso "Nuevas Tecnologías y Terapia Ocupacional". Esta encuesta fue respondida al término del curso y evaluó diversos ítems, tales como: Grado de satisfacción de los participantes con: 1) las herramientas tecnológicas usadas, 2) grado de conocimiento y disponibilidad del instructor, 3) material educativo entregado, 4) conformación de los grupos de trabajo, 5) modalidad de trabajo, 6) trabajo colaborativo, 7) grado de importancia de los temas tratados en este curso relacionados con la TO y el quehacer diario de los participantes. Estos ítems fueron evaluados por cada alumno utilizando una escala del 1 al 5 (1: muy insatisfecho; 2: insatisfecho; 3: regular; 4: satisfecho y 5: muy satisfecho).

De los datos de esta encuesta, sólo se describirán los resultados más importantes, siendo éstos los relacionados con el grado de satisfacción de los participantes con; (a) modalidad del curso on-line, (b) metodología de trabajo colaborativo del curso, (c) importancia de los temas tratados en relación al quehacer diario de los participantes y (d) grado de utilidad de las habilidades desarrolladas durante el curso en la práctica diaria de su profesión.

a) En cuanto al análisis de los resultados, se puede afirmar que la mayoría, de los participantes consideró satisfactoria la experiencia con la modalidad on-line del curso, con una respuesta de un 45\% (muy satisfactoria), un $33 \%$ (satisfactoria), y solo un $22 \%$ (regular). En relación a los resultados obtenidos en este ítem, un 22\% (regular) de los participantes mostró un bajo porcentaje de satisfacción lo cual se ve reflejado en los comentarios obtenidos de las actividades de reflexión al final de curso, entre los cuales se repitió recurrentemente que esta modalidad de trabajo carece de un formato estructurado (ej.: horarios fijos, esquema de trabajo, etc.) el cual seria un aspecto difícil de tratar a la hora de organizarse y elaborar asignaciones en conjunto.

Para ejemplificar las dificultades que tuvieron los participantes con la metodología on-line, transcribimos los siguientes comentarios:

TO (A): "No estamos acostumbrados a estudiar para la adquisición de competencias necesarias para ejercitar nuestra profesión, estamos acostumbrados a estudiar para la nota". 
TO (B): "La modalidad flexible, deja la posibilidad de aplazar el cumplimiento de asignaciones, perjudicando la constancia de trabajo grupal".

b) En cuanto a la metodología del "Aprendizaje o trabajo colaborativo", la mayoría de los participantes encontró satisfactoria su experiencia con el trabajo colaborativo desarrollado en este curso, con una respuesta del $45 \%$ (muy satisfactoria), un $11 \%$ (satisfactoria), un $33 \%$ (regular) y un $11 \%$ (insatisfecho).

c) En relación al grado de importancia que los participantes le otorgaron a los temas tratados en el curso relacionados con su quehacer diario, la mayoría de los participantes se encuentra satisfecho con éstos, teniendo una respuesta de un $45 \%$ (muy satisfecho) y un $55 \%$ (satisfecho).

d) Cabe destacar, que el mayor porcentaje de satisfacción de los participantes fue en el área del grado de utilidad encontrado a las habilidades desarrolladas en este curso en relación a la práctica diaria de su profesión, el cual alcanzó una respuesta de un 89\% (muy satisfecho) y un $11 \%$ (satisfecho).

En general, los resultados mencionados anteriormente se pueden ver reflejados en algunos comentarios de los participantes, tales como:

Estudiante (A) de TO: “La modalidad es práctica, es fácil congeniar los horarios, ya que al ser online el acceso es mayor. No te quita tanto tiempo ya que puedes hacer otras cosas a la vez".

Estudiante (B) de TO: "Me gustó la división en módulos, ya que permitió el aprendizaje gradual de las herramientas ocupadas en el curso. Me parece una buena modalidad, principalmente por la flexibilidad que otorga en cuanto al tiempo necesario y a otras situaciones no esperadas"

TO (C): "Creo que fue muy útil trabajar bajo esta modalidad, pues facilita el trabajo en equipo siendo una herramienta importante en la realización de proyectos a distancia. Si bien no ha sido fácil acostumbrarme al trabajo a distancia, creo que en la actualidad, gracias al curso, cuento con algunas herramientas que favorecen mi desempeño en esta modalidad de trabajo".

TO (D): "El haber conocido estas tecnologías realmente fue un aporte para el desarrollo de mis funciones profesionales".

Finalmente, con respecto al número de participantes, éste fue alto en la fase inicial del curso, sin embargo durante el transcurso hubo 6 deserciones $(28,5 \%)$, lo cual determinó que solo 15 $(71,5 \%)$ de los 21 alumnos continuaran su participación.

\section{Discusión}

El curso de "Nuevas Tecnologías y Terapia Ocupacional" fue la primera experiencia de un curso on-line en Chile, dirigido a profesionales y estudiantes de TO, con el propósito de explorar los usos y beneficios de las nuevas tecnologías en el quehacer tanto administrativo como educativo, de la Terapia Ocupacional. Este curso utilizó como metodología de 
enseñanza, el "Aprendizaje Colaborativo" $(21,22)$, que se destaca por tener un bajo nivel de estructuración del proceso educativo por parte del profesor y por atribuírle las responsabilidades del proceso educativo a los, los cuales deciden en grupo el nivel de profundización al cual desean llegar y el tiempo que necesitarán dedicarle a las actividades educativas.

Cabe destacar que en general este curso tuvo resultados satisfactorios en cuanto al desempeño de los grupos en las tareas asignadas, el cual alcanzó un nivel profundo de reflexión individual y grupal y un alto grado de satisfacción de los participantes con el curso. Sin embargo, la metodología de enseñanza utilizada, el "Aprendizaje Colaborativo" y la modalidad on-line, causo cierto grado de frustración e insatisfacción entre los miembros de cada uno de los grupos (Ver resultados: modalidad on-line). Es por esto que, y a modo de proponer recomendaciones para futuros cursos on-line relacionados con la TO, se analizaron los resultados de la encuesta del grado de satisfacción de los participantes junto a sus comentarios en cuanto a la metodología de enseñanza utilizada, concluyendo que la falta de estructuración del proceso educativo y la atribución de responsabilidades a los grupos para que ellos condujeran su aprendizaje, fue por una parte generadora de conflictos internos entre los alumnos.

Por otra parte, la modalidad on-line también produjo dificultades intragrupales, debido a que la mayoría de los participantes no se conocía previamente y no poder interactuar "en vivo y en directo" generó una falta de compromiso y cooperación entre ellos, provocando finalmente una desmotivación y falta de iniciativa por parte de los miembros de los grupos (Ver resultados: número de deserciones). No obstante, para futuras modalidades de cursos on-line, esto se podría evitar, fomentando más reuniones vía Skype entre los integrantes de los grupos y/o con el instructor, para que se pudieran conocer mejor entre ellos, al igual que establecer una mayor estructura en cuanto a los roles que cada miembro ejercerá dentro del grupo durante las actividades.

Sin embargo, utilizar actividades de enseñanza y aprendizaje a través de ambientes virtuales, puede generar conflictos entre los participantes, más aún si es la primera experiencia con este tipo de modalidad on-line. Por esto se aconseja a otras iniciativas que deseen utilizar estas plataformas de enseñanza virtual, plantear objetivos concretos y medibles, los cuales deberían estar relacionados con la adquisición de conocimientos y con el desarrollo de competencias, tales como, pensamiento crítico, capacidad de autorregulación del acceso al aprendizaje, solución de problemas o integración comprensiva del pensamiento, a través de un aprendizaje contextualizado y no abstracto. En nuestro caso, esto se logró a través de las características y contenidos de las asignaciones por cada módulo, las cuales se focalizaron en la generación de productos que el alumno podía utilizar posteriormente en distintas áreas del quehacer profesional.

Por otra parte, en este tipo de trabajo colaborativo hay una serie de etapas que deben considerarse:

- Delimitar el propósito del proyecto, establecer grupos de trabajo, diseñando objetivos específicos con fechas establecidas de término por cada módulo de estudio.

- El éxito o fracaso de este tipo de proyectos dependen de muchos factores, como el tipo de participantes, el grado de implicación de éstos, la cultura de aprendizaje en la que se han desenvuelto y los parámetros con los que están habituados a ser evaluados. 
- Es muy importante valorar el proceso y no solo hacer hincapié en el resultado final. Se deben considerar las competencias tecnológicas que los participantes poseen al inicio del curso para determinar conocimientos básicos en el manejo de éstas herramientas y construir el proceso educativo desde ese nivel.

- La interacción en este tipo de acciones formativas se puede establecer en diferentes niveles: entre los participantes de forma colectiva, entre el estudiante y el instructor, entre el estudiante de forma individual y entre su grupo de pares.

En resumen, se puede afirmar que estamos viviendo una nueva era de la información y la comunicación, la cual está influyendo decisivamente en las actividades de la vida diaria de las personas y en diferentes ámbitos sociales, económicos, culturales y educativos.

Internet ha impactado durante el último tiempo al constituírse en una poderosa herramienta para el intercambio y suministro de información, siendo un imperativo para los TTOO utilizar todo su potencial al servicio de los usuarios (11). Por lo mismo, se exhorta a los TTOO a conocer las nuevas tecnologías y avanzar con los tiempos contingentes (11). Tanto nuestra disciplina, como otras profesiones han demostrado, que pueden beneficiarse de las nuevas oportunidades de colaboración, retroalimentación, discusión y reflexión, que las nuevas tecnologías ofrecen.

En conclusión, las Nuevas Tecnologías, ej. Wikis, Blogs y Podcast, nos ofrecen mejorar las experiencias de aprendizaje de los estudiantes, profesionales y usuarios/clientes, profundizando los niveles de compromiso y colaboración de los participantes con el aprendizaje digital (2). Por lo tanto, se sugiere realizar futuras investigaciones para determinar la mejor manera de integrar estas herramientas en los actuales programas de aprendizaje online para los estudiantes, profesionales de la salud y usuarios/clientes, reconociendo las diferentes necesidades de cada uno de estos estamentos y la potencialidad que ofrecen las oportunidades de colaboración virtual entre ellos. De particular importancia es la investigación de éstas nuevas aplicaciones tecnológicas en educación e intervención en Terapia Ocupacional.

\section{Referencias}

1. Computacional Diccionary. Tecnologías de Información y Comunicación. [citado 16 Junio, 2011]; Disponible en: www.techterms.com/.

2. BOULOS MNK, MARAMBA I, WHEELER S. Wikis, blogs and podcasts: a new generation of Web-based tools for a virtual collaborative clinical practice and education. BMC Medical Education, 2006, 6(41), 26-31.

3. Office Online National Statistics. Use of ICT at Home 2 in 3 UK homes have digital TV service. [citado 02 Junio, 2011]; Disponible en: http://www.statistics.gov.uk/cci/nugget.asp?id=1710.

4. Development United Nations Conference on Trade and Development. Information and communication technology indicators. [citado 20 Enero 2011]; Disponible en: http://www.unctad.org/Templates/Page.asp?intItemID=5812\&lang=1. 
5. BOYD DM, ELLISON NB. Social Network Sites: Definition, History, and Scholarship. Journal of Computer-Mediated Communication 2007;13(1):210-30.

6. Statistics Facebook. [citado 05 Junio 2011]; Disponible en: www.facebook.com/press/info.php?statistics.

7. Training ECE. The use of ICT to support innovation and lifelong learning for all: A report on progress. Commission of the European Communities; 2008 [citado 16 Junio 2011]; Disponible en: http://ec.europa.eu/education/lifelong-learning-programme/doc/sec2629.pdf.

8. TOWNSEND EA. Enabling occupation: an occupational therapy perspective: Ottawa. Ont: CAOT Publications ACE.; 1991.

9. TOWNSEND EA. Enabling Occupation: an occupational therapy perspective. 2nd ed: Ottawa. Ont: CAOT Publications ACE; 2002.

10. SCHAPER LK, PERVAN GP. ICT and OTs: A model of information and communication technology acceptance and utilisation by occupational therapists. International Journal of Medical Informatics 2007;76 (Supplement 1):S212-S21.

11. BODELL S, HOOK A, PENMAN M, WADE W. Creating a learning community in today's world: how blogging can facilitate continuing professional development and international learning. British Journal of Occupational Therapy,2009, 72(6), 279-281.

12. BILLINGS D, KOWALSKI K. Online communities can provide support for nurses preparing for certification examinations. The Journal of Continuing Education in Nursing 2005; 36 (2):55-6.

13. SYKES P. Teaching and learning in Internet environments in Australian nursing education. Australia: Griffin University; 2003.

14. BLAKELY J, CURRAN-SMITH J. Teaching and community health nursing by distance methods: Development, process, and evaluation. The Journal of Continuing Education in Nursing 29(4):148-53.

15. GUNN C, MCCORMICK R, HONEY M. The challenge of change: Introducing flexible learning into a traditional medical and health sciences faculty. ASCILITE 19th annual conference - Winds of change in the sea of learning; Auckland2002.

16. O’NEILL M. Developing and implementing an online nursing course. Online Journal of Distance learning Administration 1998;1(4):1-6.

17. PLANK R. Nursing on-line for continuing education credit. The Journal of Continuing Education in Nursing 1998; 29(4):165-72.

18. HEGARTY B, STEWART A. The reality of Online Learning in nursing programmesTwo case studies. Online Learning - Scope (Flexible Learning) 2007;1:53-74.

19. HARVARD MEDICAL SCHOOL - WebWeekly [citado 02 Junio 20011] Disponible en http://webweekly.hms.harvard.edu/archive/2006/0130/student scene.html 
20. Podcasting and Vodcasting: A White Paper [citado 16 Junio 2011] Disponible en: http://edmarketing.apple.com/adcinstitute/wp-content/ Missouri_Podcasting_White_Paper.pdf

21. LANDETA AEA. E-Learning 2.0. Libro de Buenas Practicas E-Learning. Capitulo 9. [citado 02 Junio 2011]; Disponible en: http://www.buenaspracticas-elearning.com/capitulo-9e-learning-2-0.html

22. ZAÑARTU L.M. Aprendizaje colaborativo: una nueva forma de diálogo interpersonal y en Red. Revista digital de Educación y Nuevas Tecnologías Número 28 - Año V [citado 02 Junio 2011]; Disponible en: http://contexto-educativo.com.ar/2003/4/nota-02.htm).

23. SALINAS J. El aprendizaje colaborativo con los nuevos canales de comunicación, 199 227; en Cabero, J. (ed.) (2000). Nuevas tecnologías aplicadas a la educación. Madrid: Síntesis.

24. PANITZ T. \& PANITZ P. Encouraging the Use of Collaborative Learning in Higher Education. In J.J. Forest (ed.) Issues Facing International Education, June, 1998, NY, NY: Garland Publishing.

25. GROS B. El ordenador invisible. 2000. Barcelona: Gedisa.

26. PASK G. Conversation, cognition and learning. 1975. Amsterdam and New York: Elsevier.

27. VYGOTSKY L.S. El desarrollo de los procesos sicológicos superiores. 1979. Barcelona: Crítica.

28. DILLENBOURG P. What do you mean by collaborative learning?. En P. Dillenbourg 1999 (Ed) Collaborative - learning: Cognitive and Computational Approaches. 1 - 19. Oxford: Elsevier.

29. DRISCOLL M.P. \& VERGARA A. Nuevas Tecnologías y su impacto en la educación del futuro, en Pensamiento Educativo,1997; 21. 